\title{
Robotic assisted radical cystectomy: insights on long term oncological outcomes from the International Robotic Cystectomy Consortium
}

\author{
Maria Chiara Sighinolfi, ${ }^{1}$ Salvatore Micali ${ }^{1}$, Ahmed Eissa ${ }^{1,2}$, Stefano Carlo Maria Picozzi ${ }^{3}$, \\ Stefano Puliatti ${ }^{1}$, Bernardo Rocco $^{1}$ \\ ${ }^{1}$ Department of Urology, University of Modena and Reggio Emilia, Modena, Italy; ${ }^{2}$ Urology Department, Faculty of Medicine, Tanta University, \\ Tanta, Egypt; ${ }^{3}$ IRCCS Policlinico di San Donato, Milan, Italy \\ Correspondence to: Maria Chiara Sighinolfi, PhD, MD. Department of Urology, University of Modena and Reggio Emilia, Via del Pozzo 71 , 41100 \\ Modena, Italy. Email: sighinolfic@yahoo.com. \\ Provenance: This is an invited article commissioned by the Section Editor Dr. Xiao Li (Department of Urology, Jiangsu Cancer Hospital \& Jiangsu \\ Institute of Cancer Research \& Affiliated Cancer Hospital of Nanjing Medical University, Nanjing, China). \\ Comment on: Hussein AA, Elsayed AS, Aldhaam NA, et al. Ten-Year Oncologic Outcomes Following Robot-Assisted Radical Cystectomy: Results \\ from the International Robotic Cystectomy Consortium. J Urol 2019;202:927-35.
}

Submitted Aug 18, 2019. Accepted for publication Dec 05, 2019.

doi: $10.21037 /$ tau.2019.12.05

View this article at: http://dx.doi.org/10.21037/tau.2019.12.05

The goal of minimally invasive surgery in the field of bladder cancer (BCa) is to overcome the complexity of the open approach, to minimize surgical morbidity and to improve recovery. Apart from a brief phase attempting a pure laparoscopic approach, the steep and exhaustive learning curve (LC) and the difficulty of radical cystectomy (RC) made the trend rapingly moving toward the robotic assistance (1). The introduction of robotic assisted radical cystectomy (RARC) was initially slow, but afterwards it steadily increased both in USA and in Europe (1). In 12 tertiary referral centers-whose experience has been collected in a multicenter collaboration study from Zamboni et al.- -the frequency of RARC has overcome the one of open RC in the $2015-2018$ period (54\% vs. $46 \%$, respectively) (1). Notwithstanding some differences—such as major use of neobladder in European countries-the global tendency is similar, with blood loss and length of hospital stay favoring the robotic approach $(2,3)$. Apart from short-term benefits of RARC, some previous oncological concerns-as spread and dissemination of neoplastic cells, atypical sites of recurrence-have been limiting some surgeons technically willing to afford the shift toward robotics for RC. The article from Hussein et al. finally addresses the issue of RARC safety, providing the largest series ever published on oncological outcomes evaluated
10 years after surgery (4).

Data were retrieved from the International Robotic Cystectomy Consortium (IRCC) dataset; IRCC was founded in 2006 and consists of a mixture of academic and private centers, who joined their RARC results in a pooled database enrolling cases since 2003. The aim was to create a collaborative venture to better understand the insights of the new operative modality (5).

A total of 446 patients who underwent RARC up to 2009 were considered from IRCC database (4). Despite the very beginning of the robotic era, the case series seems not to be biased by patients' selection, since locally advanced diseases ( $\geq$ pT3) finally accounted for $43 \%$, with overall nodal involvement in $24 \%$. Most of the cases were performed with an extracorporeal diversion, reflecting an initial cohort; thought, some of the more recent series or RCTs-as the RAZOR one (2) -still continue to rely on an extracorporeal approach too, possibly limiting the advantages of RARC in the perioperative setting.

From their initial IRCC dataset, Hussein et al. (4) analyzed major oncological endpoints at 10 yearsspecifically recurrence free (RFS), disease-specific (DSS) and overall survival (OS) - and found 59\% of RFS, $65 \%$ of DSS and $35 \%$ of OS. As stated, they appeared similar to the ones reported historically for open surgery. Overall, 
$44 \%$ of patients had their relapse within the 5 -year period, consistent with the finding that most $\mathrm{BCa}$ recurrences manifest within first years after surgery. Local recurrences were reported in $15 \%$ of patients, extra-pelvic nodal in $5 \%$, abdominal wall metastasis and peritoneal carcinomatosis in $2.4 \%$. No port-site recurrences were noted. To summarize, long-term data seem to support the introduction of RARC, which should be regarded as a safe procedure with enduring oncological results.

Nevertheless, this initial series may suffer from a selection bias-not involving patients'-but surgeons' selection. Even if IRCC aimed to depict RARC during its evolution and technical standardization (5), it should be kept in mind that most of the centers already had high volumes and previous laparoscopic and/or robotic expertise. Before entering IRCC trial and database, more than a third of surgeons have performed $\geq 20$ RARC (6). An interim IRCC analysis published in 2010 highlighted that more than $80 \%$ of RARC before 2009 have been performed by surgeons already skilled in robotic radical prostatectomy ( $>50$ cases) (7). Whether the burden of - and how-a previous RALP series may endorse RARC is unclear, but we have to acknowledge that many IRCC surgeons were already accustomed to robotic approach to the pelvis and to principles of laparoscopy and/or robotics.

Such a comment arises from two matters: first, the positive margin rate at soft specimen is $7 \%$, fairly low especially if related to the high rate of locally advanced disease ( $43 \% \geq \mathrm{pT} 3$ ) (4); second, the routinely use of pelvic lymphadenectomy (PLND) was not so common at that time, thus indicating the compliance to good oncological practice with adequate surgical skills among IRCC surgeons (6). A study from Hellenthal et al. found that PLND was accomplished only in $30-40 \%$ of RC by the end of the previous decade (8). The same author in 2010in a paper on PLND in the initial IRCC series-stated that "participating (IRCC) surgeons have some impetus to improve individual outcomes" and performed interventions at their best (6). This evidence may definitely result in short-term (PSM) and mid-term (RFS) excellent results from the initial IRCC team, resembling the ones reached from RCTs and bigger series a decade afterwards.

Margin status and nodal yield-from the surgeons' perspective-are surrogates of good surgical quality for most of the oncological procedures. But a PSM, especially in the field of $\mathrm{BCa}$, is also a surrogate of adverse oncological course, as it affects local recurrence, it doubles the metastatic progression risk, and it impairs cancer specific survival (9-11).

As expected, also in the current IRCC series, PSM is related to RFS. Remarkably, biological behavior of pT2 and of non-muscle invasive $\mathrm{BCa}$ is similar in terms of RFS and DSS, suggesting low or absent PSMs for organ-confined disease (4). The chance to have also a locally advanced disease completely and radically removed could translate into a better RFS, DSS and overall oncological endpoints improved.

Advancement and aggressiveness of $\mathrm{BCa}-$ i.e., high pT, lymphovascular and nodal invasion - have been invoked as main drivers of adverse prognosis in both clinical and experimental settings $(12,13)$. The recent article by Wei et al. (12) raises the concerns of residual cancer cells persisting in washing specimen after RARC; the way these cells are spread into peritoneum is still uncertain, but cancer aggressiveness seems to be the main driver of this kind of microscopical persistence $(12,14)$.

Biological behavior of transitional cell carcinoma is far to be defined: in this uncertain setting, the basic rules of surgical oncology-complete removal of the specimen when affordable-together with principles restored for laparoscopy (15)—namely a proper manipulation of the cystectomy specimen, the avoidance of inadvertent dissection into bladder or nodes, the avoidance of breach of the specimen bag, together with a careful prevention of urinary spillage from the ureters or urethral stump-still continue to be crucial.

What lesson can be learned from the paper by Hussein et al. (4)? Perioperative and oncological outcomes from the IRCC are actually reproducible and transferrable elsewhere? On one hand, the paper ensures urologists about a feasible, fast and safe transition from open to robotic RC; but, on the other, surgeons' volume and a high-level robotic expertise seems to be of paramount importance to address the complexity of RARC and to control the aggressiveness of urothelial cancer in the laparoscopic environment.

\section{Acknowledgments}

None.

\section{Footnote}

Conflicts of Interest: The authors have no conflicts of interest to declare. 
Ethical Statement: The authors confirms that they are accountable for all aspects of the work in ensuring that questions related to the accuracy or integrity of any part of the work are appropriately investigated and resolved.

\section{References}

1. Zamboni S, Soria F, Mathieu R, et al. Differences in trends in the use of robot-assisted and open radical cystectomy and changes over time in peri-operative outcomes among selected centres in North America and Europe: an international multicentre collaboration. BJU Int 2019. DOI: 10.1111/bju.14791.

2. Parekh DJ, Reis IM, Castle EP, et al. Robot-assisted radical cystectomy versus open radical cystectomy in patients with bladder cancer (RAZOR): an open-label, randomised, phase 3, non-inferiority trial. Lancet 2018;391:2525-36.

3. Rai BP, Bondad J, Vasdev N, et al. Robotic versus open radical cystectomy for bladder cancer in adults. Cochrane Database Syst Rev 2019;4:CD011903.

4. Hussein AA, Elsayed AS, Aldhaam NA, et al. Comparison of Long-Term Oncologic Outcomes among Historical Open and Minimally Invasive Retrospective Studies. J Urol 2019;202:927-35.

5. Raza SJ, Field E, Kibel AS, et al. International Robotic Radical Cystectomy Consortium: A way forward. Indian J Urol 2014;30:314-7.

6. Hellenthal NJ, Hussain A, Andrews PE, et al. Lymphadenectomy at the time of robot-assisted radical cystectomy: results from the International Robotic Cystectomy Consortium. BJU Int 2011;107:642-6.

7. Hayn MH, Hellenthal NJ, Hussain A, et al. Does previous robot-assisted radical prostatectomy experience affect

Cite this article as: Sighinolfi MC, Micali S, Eissa A, Picozz SCM, Puliatti S, Rocco B. Robotic assisted radical cystectomy: insights on long term oncological outcomes from the International Robotic Cystectomy Consortium. Transl Androl Urol 2019;8(Suppl 5):S521-S523. doi: 10.21037/tau.2019.12.05 outcomes at robot-assisted radical cystectomy? Results from the International Robotic Cystectomy Consortium. Urology 2010;76:1111-6.

8. Hellenthal NJ, Ramirez ML, Evans CP, et al. Trends in pelvic lymphadenectomy at the time of radical cystectomy: 1988 to 2004. J Urol 2009;181:2490-5.

9. Bochner BH, Dalbagni G, Sjoberg DD, et al. Comparing Open Radical Cystectomy and Robot-assisted Laparoscopic Radical Cystectomy: A Randomized Clinical Trial. Eur Urol 2015;67:1042-50.

10. Dotan ZA, Kavanagh K, Yossepowitch O, et al. Positive surgical margins in soft tissue following radical cystectomy for bladder cancer and cancer specific survival. J Urol 2007;178:2308-12; discussion 2313.

11. Herr HW, Faulkner JR, Grossman HB, et al. Surgical factors influence bladder cancer outcomes: a cooperative group report. J Clin Oncol 2004;22:2781-9.

12. Wei L, Hussein AA, Ma Y, et al. Accurate Quantification of Residual Cancer Cells in Pelvic Washing Reveals Association with Cancer Recurrence Following RobotAssisted Radical Cystectomy. J Urol 2019;201:1105-14.

13. Hussein AA, Saar M, May PR, et al. Early Oncologic Failure after Robot-Assisted Radical Cystectomy: Results from the International Robotic Cystectomy Consortium. J Urol 2017;197:1427-36.

14. Sighinolfi MC, Rocco B, Micali S, et al. Re: Accurate Quantification of Residual Cancer Cells in Pelvic Washing Reveals Association with Cancer Recurrence following Robot-Assisted Radical Cystectomy. J Urol 2019 ;202:1271-2.

15. Sighinolfi MC, Micali S, Celia A, et al. Laparoscopic radical cystectomy: an Italian survey. Surg Endosc 2007;21:1308-11. 